\title{
RACK1 silencing attenuates renal fibrosis by inhibiting TGF- $\beta$ signaling
}

\author{
JIE FENG $^{1}$, LIYI XIE ${ }^{1}$, RANRAN KONG $^{2}$, YALI ZHANG ${ }^{1}$, KEHUI SHI $^{3}$, WANHONG LU ${ }^{1}$ and HONGLI JIANG ${ }^{3}$ \\ ${ }^{1}$ Department of Nephrology, The First Affiliated Hospital of Xi'an Jiaotong University, Xi'an, Shaanxi 710061; \\ ${ }^{2}$ Department of Thoracic Surgery, The Second Affiliated Hospital of Xi'an Jiaotong University, Xi'an, Shaanxi 710004; \\ ${ }^{3}$ Blood Purification Center, The First Affiliated Hospital of Xi'an Jiaotong University, Xi'an, Shaanxi 710061, P.R. China
}

Received January 6, 2016; Accepted September 18, 2017

DOI: $10.3892 /$ ijmm.2017.3154

\begin{abstract}
The receptor for activated C-kinase 1 (RACK1) is a member of the WD40-repeat family of proteins and has been reported to be implicated in the development of liver fibrosis. However, the role of RACK1 in renal fibrosis remains unclear. Therefore, in this study, we investigated the effects of RACK1 on transforming growth factor- $\beta 1$ (TGF- $\beta 1$ )-treated human proximal tubular epithelial cells and aimed to elucidate the possible mechanisms responsible for its anti-fibrotic effects. Our results revealed that RACK1 was highly expressed in the renal fibrotic tissues and TGF- $\beta 1$-treated HK- 2 cells. RACK1 silencing inhibited TGF- $\beta 1$-induced $\alpha$-smooth muscle actin and connective tissue growth factor expression in the HK-2 cells. Furthermore, RACK1 silencing inhibited the expression of phosphorylated Smad3 in the TGF- $\beta 1$-treated HK-2 cells. To the best of our knowledge, these data demonstrate for the first time the role of RACK1 in renal fibrosis. The present findings indicate that RACK1 silencing attenuates renal fibrosis by suppressing the activation of TGF- $\beta 1 / \mathrm{Smad} 3$ signaling pathway in HK-2 cells. Thus, RACK1 may serve as a novel regulator of renal fibrosis.
\end{abstract}

\section{Introduction}

Renal fibrosis is a common condition which can lead to various types of progressive chronic kidney disease and even end-stage renal failure. It is characterized by the activation of renal fibroblasts and the accumulation of excessive amounts of extracellular matrix $(\mathrm{ECM})$ proteins $(1,2)$. Transforming growth factor- $\beta 1$ (TGF- $\beta 1$ ) is the most important growth factor which regulates the transdifferentiation of tubular epithelial cells

Correspondence to: Dr Hongli Jiang, Blood Purification Center, The First Affiliated Hospital of Xi'an Jiaotong University, 277 Yanta West Road, Xi'an, Shaanxi 710061, P.R. China

Email: jhonglibpc@sina.com

Key words: receptor for activated C-kinase 1, renal fibrosis, transforming growth factor- $\beta 1$ into myofibroblasts in renal fibrosis (3). Previous studies have demonstrated that TGF- $\beta 1$ expression is markedly elevated in animal models of renal fibrosis and in patients with glomerulonephritis $(4,5)$. Thus, the suppression of TGF- $\beta 1$ signaling may be a potential target with which to prevent progressive renal fibrosis.

WD40-repeat proteins are a large, highly conserved family of adaptors implicated in various biological process, such as signal transduction, gene transcriptional regulation, protein modifications, cytoskeleton assembly, vesicular trafficking, DNA damage and repair, cell death and cell cycle progression (6). The receptor for activated C-kinase 1 (RACK1) is a member of the WD40-repeat family of proteins and has been reported to be implicated in the development of various diseases (7-9). A previous study implied that RACK1 overexpression inhibited cardiomyocyte apoptosis following myocardial ischemia/reperfusion in adult rats (10). The downregulation of RACK1 has been shown to inhibit cell proliferation, along with invasion and migration in vitro and in vivo in esophageal squamous cell carcinoma (11). Moreover, a previous study reported that RACK 1 promoted the TGF- $\beta 1$ mediated activation of pro-fibrogenic pathways, as well as the differentiation, proliferation and migration of hepatic stellate cells (HSCs), and the depletion of RACK1 suppressed the progression of thioacetamide-induced liver fibrosis in vivo (12). However, the role of RACK1 in renal fibrosis remains unclear. Therefore, in this study, we investigated the effects of RACK1 on TGF- $\beta 1$-treated human proximal tubular epithelial cells and aimed to elucidate the possible mechanisms responsible for its anti-fibrotic effects.

\section{Materials and methods}

Specimen collection. Renal biopsy samples were collected by transparietal puncture from 12 healthy individuals and 10 patients with renal fibrosis, diagnosed on clinical, biological and histological grounds. The samples were immediately stored in liquid nitrogen in preparation for use. This study was approved by the Ethics Committee of the First Affiliated Hospital of Xi'an Jiaotong University (Xi'an, China), and written informed consent was obtained from all participants prior to sample collection. 
Cell culture and treatment. The human proximal tubular epithelial cell line, HK-2, was purchased from the American Type Culture Collection (ATCC; Manassas, VA, USA). The HK-2 cells were maintained in Dulbecco's modified Eagle's medium (DMEM) supplemented with $5 \%$ fetal bovine serum (FBS) (both from Invitrogen Life Technologies, Carlsbad, CA, USA), $100 \mathrm{U} / \mathrm{ml}$ penicillin and $100 \mathrm{mg} / \mathrm{ml}$ streptomycin (Sigma-Aldrich, St. Louis, MO, USA) at $37^{\circ} \mathrm{C}$ in a humidified $5 \% \mathrm{CO}_{2}$ atmosphere. The $\mathrm{HK}-2$ cells were seeded in the complete medium containing 5\% FBS at approximately $70 \%$ confluence in 6-well culture plates. After $24 \mathrm{~h}$, the complete medium was replaced with serum-free medium for $24 \mathrm{~h}$ prior to treatment with recombinant TGF- $\beta 1$ ( $5 \mathrm{ng} / \mathrm{ml}$; Sigma-Aldrich).

Small interfering RNA (siRNA) transfection. siRNA targeting RACK1 or its corresponding negative control was designed and synthesized from Sangon Biotech Co., Ltd. (Shanghai, China). For in vitro transfection, the HK-2 cells were plated and grown to 70-90\% confluency without antibiotics, and then transfected with siRNA-RACK1 or siRNA-mock using Lipofectamine ${ }^{\mathrm{TM}} 2000$ (Invitrogen Life Technologies) according to the manufacturer's instructions. The transfection efficiency was evaluated by examining RACK1 mRNA and protein expression by reverse transcription-quantitative polymerase chain reaction (RT-qPCR) and western blot analysis, respectively.

RT-qPCR analysis. Total RNA was extracted from the HK-2 cells using TRIzol reagent (Takara Biotechnology Co., Ltd., Dalian, China). Approximately $5 \mu \mathrm{g}$ total RNA from each sample was reverse transcribed into cDNA using SuperScript II Reverse Transcriptase (Takara Biotechnology Co., Ltd.). Quantitative (real-time) PCR was performed on an ABI-Prism 7500 using Power SYBR-Green Master Mix 2X (Applied Biosystems, Foster City, CA, USA). The following primers were used: RACK1, 5'-TTC TCC TCT GAC AAC CGG CA-3' (sense), 5'-GCC ATC CTT GCC TCC AGA A-3' (antisense); $\alpha$-smooth muscle actin ( $\alpha$-SMA), 5'-CTA TTC CTT CGT GAC TAC T-3' (sense), 5'-ATG CTG TTA TAG GTG GTG GTT-3' (antisense); connective tissue growth factor (CTGF), 5'-GGA AAA GAT TCC CAC CCA AT-3' (sense), 5'-TGC TCC TAA AGC CAC ACC TT (antisense); and $\beta$-actin, 5'-GGC AAA TTC AAC GGC ACA GTC-3' (sense), 5'-GCT GAC AAT CTT GAG TGA GTT-3' (antisense). The cycling conditions were as follows: pre-incubation at $95^{\circ} \mathrm{C}, 10 \mathrm{~min}$; PCR: $95^{\circ} \mathrm{C}, 15 \mathrm{sec}$ and $59^{\circ} \mathrm{C}, 30 \mathrm{sec}, 35$ cycles; final elongation: $72^{\circ} \mathrm{C}, 10 \mathrm{~min}$. $\beta$-actin was used as an internal control and the expression levels of the relative genes were calculated using the $2^{-\Delta \Delta \mathrm{CT}}$ method.

Western blot analysis. Total protein was extracted from the HK-2 cells, then washed with ice-cold PBS and lysed with RIPA cell lysis buffer (Cell Signaling Technology, Inc., Danvers, MA, USA) containing a phosphatase inhibitor and the protease inhibitor cocktail (Sigma-Aldrich), by incubating on ice for $30 \mathrm{~min}$. The protein concentration was assayed using a micro BCA protein kit (Pierce, Rockford, IL, USA). Forty micrograms of protein per lane were separated by $10 \%$ SDS-PAGE and then transferred onto polyvinylidene fluoride (PVDF) membranes (Millipore, Boston, MA, USA). Non-specific binding sites were blocked with 5\% (w/v) skim milk (Bio-Rad Laboratories, Inc., Hercules, CA, USA) in Tris-buffered saline-Tween-20 (TBS-T) for $1 \mathrm{~h}$ at room temperature and the membranes were then incubated with anti-RACK1 (1:1,000 dilution; ab62735), anti- $\alpha$-SMA (1:2,500 dilution; ab7817), anti-CTGF (1:1,000 dilution; ab94939), anti-p-Smad3 (1:2,500 dilution; ab51177), (all from Abcam, Cambridge, UK) anti-Smad3 (1:1,000 dilution; sc-101154) and anti-GAPDH (1:1,500 dilution; sc-47724) (all from Santa Cruz Biotechnology, Inc., Santa Cruz, CA, USA) antibodies at $4^{\circ} \mathrm{C}$ overnight. The membranes were then washed and incubated with horseradish peroxidase-conjugated secondary antibodies (sc-2005 and sc-2004; Santa Cruz Biotechnology, Inc.). The absorbance values of the target proteins were determined using Gel-Pro Analyzer version 4.0 software (Media Cybernetics, Silver Spring, MD, USA).

Statistical analysis. All data are presented as the means \pm standard deviation (SD) based on at least 3 independent experiments. Statistical analysis was performed using the Student's t-test and ANOVA. A value of $\mathrm{P}<0.05$ was considered to indicate a statistically significant difference compared to the respective control.

\section{Results}

RACK1 is highly expressed in renal fibrotic tissues. In order to examine the expression of RACK1 in renal fibrosis, the mRNA transcription level of RACK1 was examined by RT-qPCR. As shown in Fig. 1A, RACK1 mRNA expression was markedly increased in the renal fibrotic tissues, as compared with those of the control group. Similarly, western blot analysis demonstrated that the protein expression of RACK1 was also upregulated in the renal fibrotic tissues (Fig. 1B).

RACK1 expression is rapidly induced in TGF- $\beta 1$-treated $H K-2$ cells. We then examined the expression of RACK1 in TGF- $\beta 1$-treated HK-2 cells. As indicated in Fig. 2, the results revealed that TGF- $\beta 1$ treatment significantly upregulated the mRNA and protein expression of RACK1 in a time-dependent manner.

RACK1 silencing inhibits TGF- $\beta 1$-induced $\alpha$-SMA and CTGF in HK-2 cells. To confirm the role of RACK1 in TGF- $\beta 1-$ induced renal fibrogenesis, we used specific siRNA to knock down the expression of RACK1. As shown in Fig. 3, the RACK1 mRNA and protein expression levels in the siRNA-RACK1transfected group were significantly lower than those in the siRNA-mock transfected group. We then evaluated the effects of siRNA-RACK 1 on the $\alpha$-SMA and CTGF expression levels in the TGF- $\beta 1$-treated HK-2 cells. As shown in Fig. 4A and C, TGF- $\beta 1$ treatment greatly increased the expression of $\alpha$-SMA at both the mRNA and protein level, while the knockdown of RACK1 inhibited the expression of $\alpha$-SMA in the TGF- $\beta 1$ treated HK-2 cells. Similarly, RACK1 silencing also suppressed the TGF- $\beta 1$-induced increase in CTGF expression in the HK-2 cells (Fig. 4A and C).

RACK1 silencing inhibits the expression of phosphorylated Smad3 in TGF- $\beta 1$-treated HK-2 cells. Smad3 is a critical transcription factor that mediates cellular fibrotic response to TGF- $\beta 1$ (13). Therefore, in this study, we examined the 


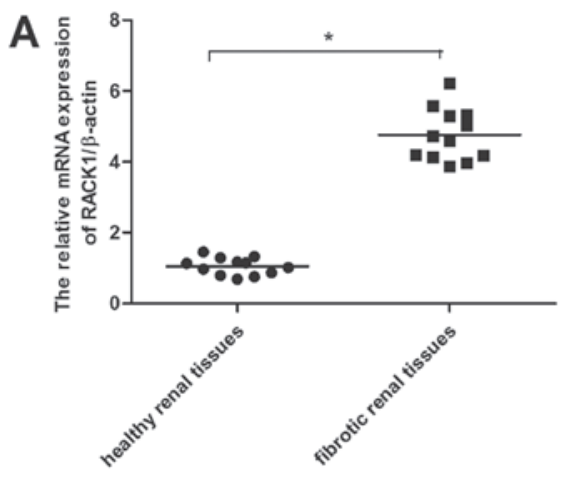

B

RACK1

GAPDH

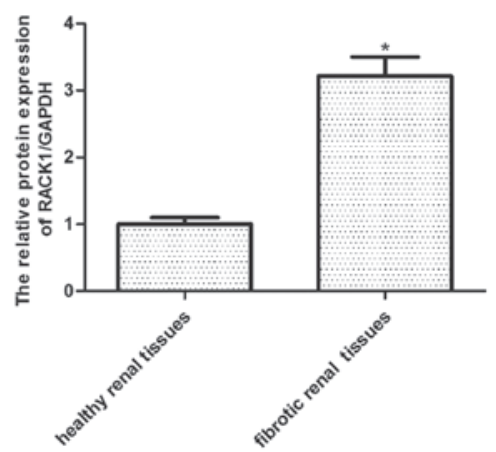

Figure 1. Receptor for activated C-kinase 1 (RACK1) is highly expressed in renal fibrotic tissues. (A) RT-qPCR and (B) western blot analysis of RACK1 expression in renal fibrotic tissues. In the blots, the lanes (left to right) indicate healthy renal tissue and fibrotic tissue, respectively. Data are expressed as the means \pm SD. Experiments were performed in triplicate. ${ }^{*} \mathrm{P}<0.05$ compared with the healthy renal tissues.
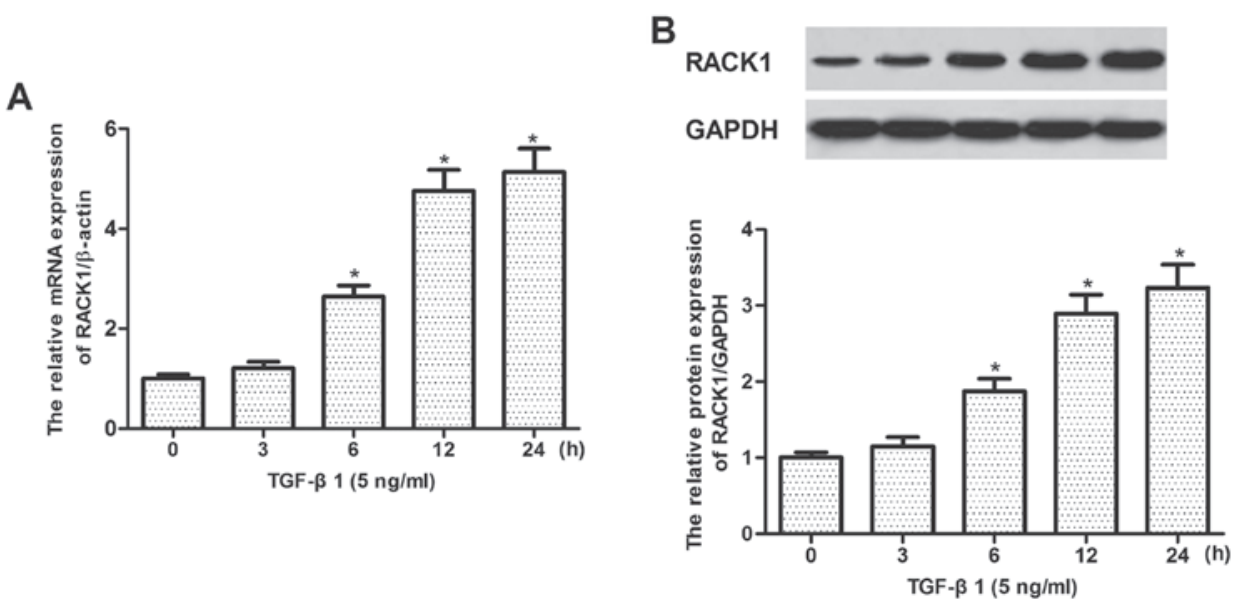

Figure 2. Receptor for activated C-kinase 1 (RACK1) expression is rapidly induced in transforming growth factor- $\beta 1$ (TGF- $\beta 1$ )-treated HK-2 cells. HK-2 cells were maintained in the absence or presence of TGF- $\beta 1(5 \mathrm{ng} / \mathrm{ml})$ for various periods of time. (A) RT-qPCR and (B) western blot analysis of RACK1 expression in HK-2 cells. In the blots, the lanes (left to right) indicate the 0-24 h time points, respectively. Data are expressed as the means \pm SD. Experiments were performed in triplicate. $\mathrm{P}<0.05$ compared with the control group.

\section{A}

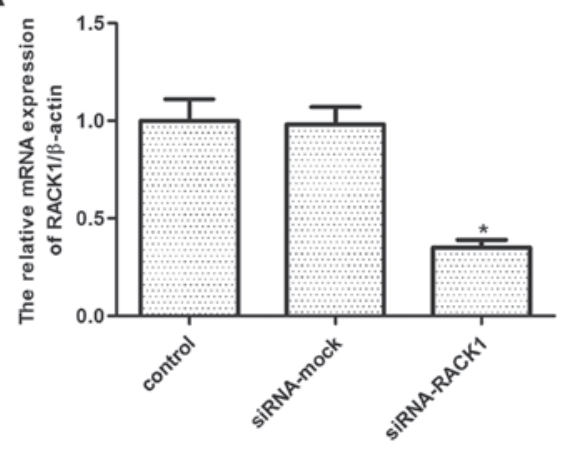

B

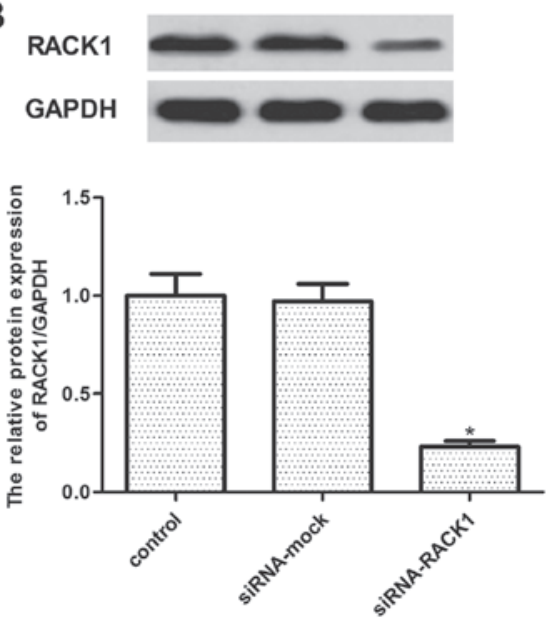

Figure 3. The expression of receptor for activated C-kinase 1 (RACK1) is significantly decreased in small interfering RNA (siRNA)-RACK1-transfected HK-2 cells. (A) RT-qPCR analysis of RACK1 mRNA expression in siRNA-mock and siRNA-RACK1 transfected HK-2 cells. (B) Western blot analysis of RACK1 protein expression in siRNA-mock and siRNA-RACK1 transfected HK-2 cells. In the blots, the lanes (left to right) indicate the control, mock siRNA group and the RACK1 siRNA group, respectively. Data are expressed as the means \pm SD. Experiments were performed in triplicate. "P<0.05 compared with the siRNA-mock group. 


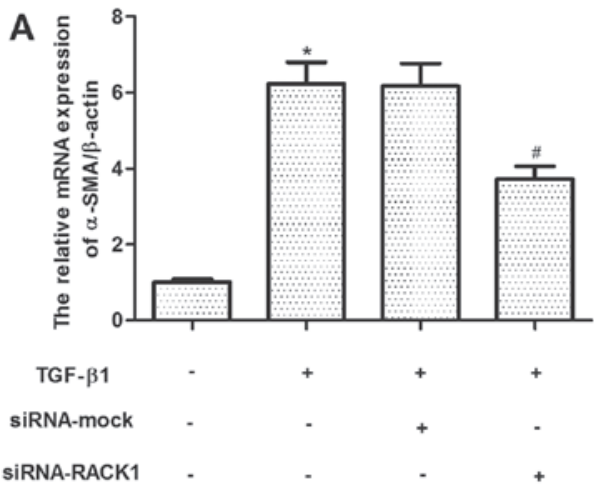

C

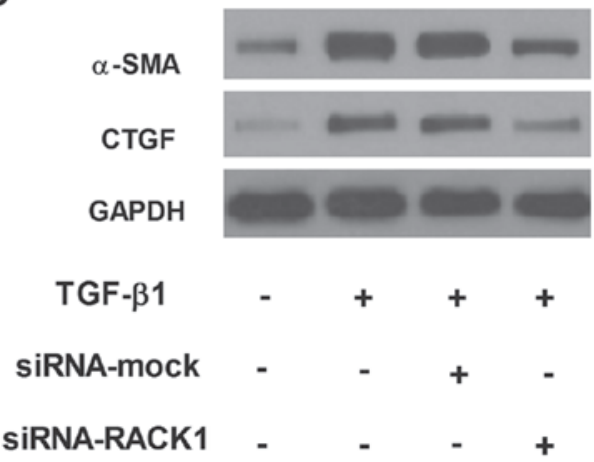

B

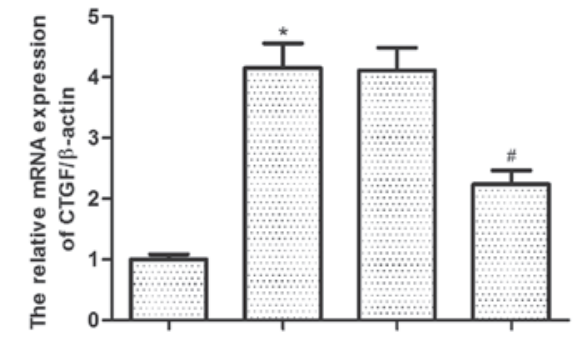

TGF- $\beta 1$

SIRNA-mock

SIRNA-RACK1

D

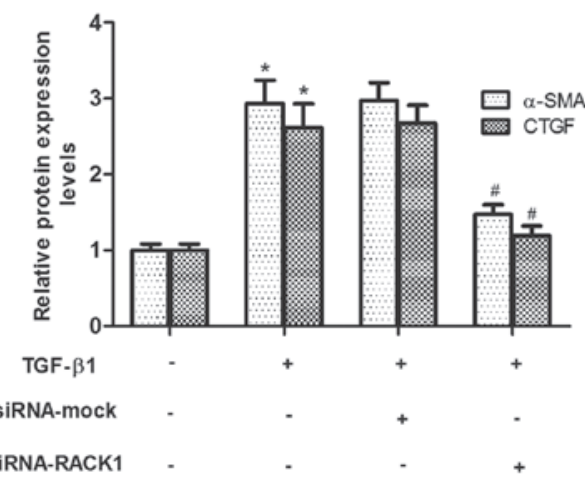

Figure 4. Receptor for activated C-kinase 1 (RACK1) silencing inhibits transforming growth factor- $\beta 1$ (TGF- $\beta 1$ )-induced $\alpha$-smooth muscle actin ( $\alpha$-SMA) and connective tissue growth factor (CTGF) expression in HK-2 cells. HK-2 cells were transfected with small interfering RNA (siRNA)-mock or siRNA-RACK1 and serum-starved overnight, after which they were stimulated with TGF- $\beta 1$ for $24 \mathrm{~h}$. (A and B) The mRNA expression levels of $\alpha$-SMA and CTGF were determined by RT-qPCR. (C) The protein expression levels of $\alpha$-SMA and CTGF were determined by western blot analysis. (D) The relative protein expression levels of $\alpha$-SMA and CTGF were quantified using Gel-Pro Analyzer version 4.0 software and normalized to GAPDH. Data are expressed as the means \pm SD. Experiments were performed in triplicate. ${ }^{*} \mathrm{P}<0.05$ compared with the control group; ${ }^{*} \mathrm{P}<0.05$ compared with the siRNA-mock group.

A

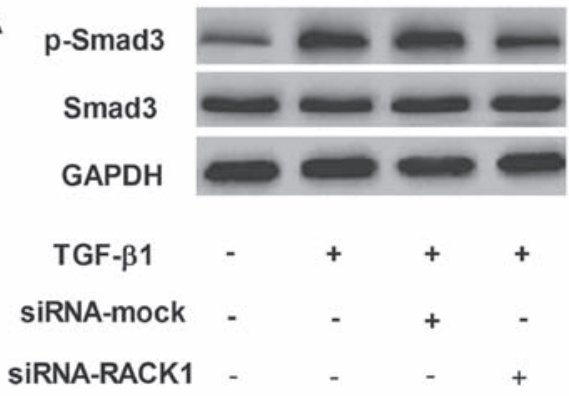

B

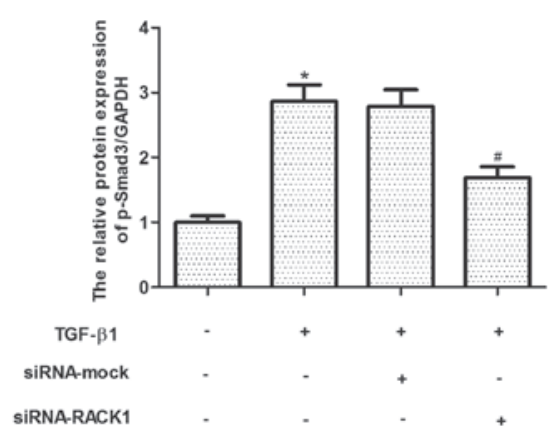

C

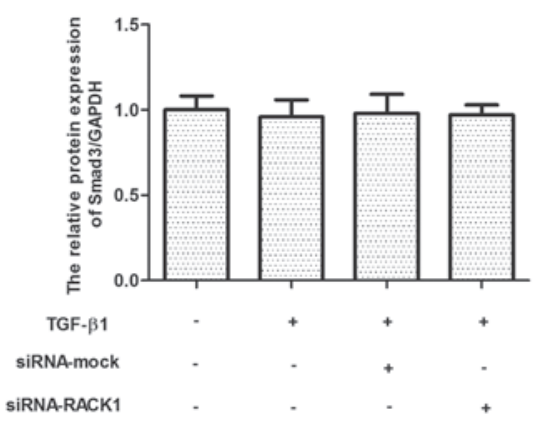

Figure 5. Receptor for activated C-kinase 1 (RACK1) silencing inhibits the expression of phosphorylated Smad3 in transforming growth factor- $\beta 1$ (TGF- $\beta 1$ )-treated HK-2 cells. HK-2 cells were transfected with small interfering RNA (siRNA)-mock or siRNA-RACK1 and serum-starved overnight, after which they were stimulated with TGF- $\beta 1$ for $1 \mathrm{~h}$. (A and B) The protein expression levels of $\mathrm{p}-\mathrm{Smad} 3$ and $\mathrm{Smad} 3$ were determined by western blot analysis. (B) The relative protein expression levels of p-Smad3 and (C) Smad3 were quantified using Gel-Pro Analyzer version 4.0 software and normalized to GAPDH. Data are expressed as the means \pm SD. Experiments were performed in triplicate. ${ }^{*} \mathrm{P}<0.05$ compared with the control group; ${ }^{\prime \prime} \mathrm{P}<0.05$ compared with the siRNA-mock group.

effect of RACK1 on the expression of p-Smad3 and Smad3 in TGF- $\beta 1$-treated HK- 2 cells. As shown in Fig. 5, compared with the control, TGF- $\beta 1$ treatment markedly increased the

expression of phosphorylated Smad3, while the knockdown of RACK1 inhibited the expression of phosphorylated Smad3 in the TGF- $\beta 1$-treated HK- 2 cells. The silencing of RACK1 had no effect on the expression of Smad3. 


\section{Discussion}

The main findings of the present study were the following: i) RACK1 was highly expressed in renal fibrotic tissues and TGF- $\beta 1$-treated HK-2 cells; ii) RACK1 silencing inhibited the TGF- $\beta 1$-induced increase in the expression of $\alpha$-SMA and CTGF in HK-2 cells; iii) RACK1 silencing inhibited the expression of phosphorylated Smad3 in the TGF- $\beta 1$-treated HK-2 cells. To the best of our knowledge, these data demonstrate for the first time the role of RACK1 in renal fibrosis.

A previous study demonstrated that the expression of RACK1 was increased in activated HSCs (12). However, RACK1 was also found to be involved in dermal fibrosis, and the expression of RACK1 was found to be significantly decreased in keloid fibroblasts (14). Therefore, RACK1 has a pro-fibrogenic or antifibrogenic function depending on the cell type or context. In the present study, we observed that RACK1 was highly expressed in renal fibrotic tissues and TGF- $\beta 1$-treated HK-2 cells. These findings suggest that RACK1 may have a pro-fibrogenic function in the development of renal fibrosis.

A growing body of evidence suggests that the ECM plays an important role in the pathogenesis of renal fibrosis. CTGF acts as a downstream target of pro-fibrotic genes and is involved in the formation of the ECM proteins, fibronectin and collagen $(15,16)$. Its expression is considered a molecular hallmark of renal fibrosis. Yokoi et al reported that CTGF antisense treatment significantly inhibited the induction of CTGF and ECM genes and reduced renal fibrotic area in rat obstructive nephropathy (17). In epithelial-mesenchymal transition (EMT), the loss of tubular epithelial cell adhesion molecules, such as E-cadherin, is replaced by $\alpha$-SMA, which is one of the interstitial myofibroblast activation markers (18). In addition, TGF- $\beta 1$ can induce $\alpha$-SMA expression and decreases adhesive protein expression in human renal tubular epithelia cells (19). In line with these results, herein, we observed that TGF- $\beta 1$ induced the expression of $\alpha$-SMA and CTGF in HK- 2 cells, while RACK1 silencing inhibited the TGF- $\beta 1$-induced increase in the expression of $\alpha$-SMA and CTGF in HK- 2 cells. These results suggest that RACK1 silencing markedly attenuated renal fibrosis via the reduction of ECM expression in TGF- $\beta 1$-stimulated HK-2 cells.

There is evidence to indicate that the TGF- $\beta 1 / \mathrm{Smad}$ signaling pathway plays a critical role in the development of renal fibrosis (20-23). During fibrogenesis, the activated type I receptor kinase phosphorylates Smad2 and 3, which forms an oligomeric complex with Smad4 that translocates into the nucleus to regulate the transcription of target genes in collaboration with various co-activators and co-repressors (24). In chronic kidney disease, TGF- $\beta$ overexpression induces renal fibrosis, while TGF- $\beta$ inhibition suppresses the expression of TGF- $\beta 1$ and type I collagen in tubulointerstitial cells in unilateral ureteral obstruction (UUO)induced renal fibrosis $(25,26)$. Phosphorylated Smad3 is highly expressed in the obstructed kidney (27), and Smad3 promotes renal fibrosis by directly binding to the promoter region of collagens to trigger their production (28), while the deletion of Smad3 inhibits fibrogenesis in a number of rodent models and the inhibition of Smad3 prevented endothelial-myofibroblast transition and renal fibrosis in type-1 diabetic kidney disease (29). In the present study, we found that RACK1 silencing inhibited the expression of phosphorylated Smad3 in TGF- $\beta 1$-treated HK-2 cells. These results suggest that RACK1 silencing attenuates renal fibrosis by suppressing the activation of the TGF- $\beta 1 / \mathrm{Smad} 3$ signaling pathway in HK-2 cells.

In conclusion, the present findings indicate that RACK1 silencing attenuates renal fibrosis by suppressing the activation of the TGF- $\beta 1 / \mathrm{Smad} 3$ signaling pathway in HK-2 cells. Thus, RACK1 may serve as a novel regulator of renal fibrosis.

\section{References}

1. Boor P, Ostendorf T and Floege J: Renal fibrosis: novel insights into mechanisms and therapeutic targets. Nat Rev Nephrol 6: 643-656, 2010.

2. Eddy AA: Molecular basis of renal fibrosis. Pediatr Nephrol 15: 290-301, 2000.

3. Stahl PJ and Felsen D: Transforming growth factor- $\beta$, basement membrane, and epithelial-mesenchymal transdifferentiation: implications for fibrosis in kidney disease. Am J Pathol 159: 1187-1192, 2001

4. López-Hernández FJ and López-Novoa JM: Role of TGF- $\beta$ in chronic kidney disease: an integration of tubular, glomerular and vascular effects. Cell Tissue Res 347: 141-154, 2012.

5. Mozes MM, Böttinger EP, Jacot TA and Kopp JB: Renal expression of fibrotic matrix proteins and of transforming growth factor- $\beta$ (TGF- $\beta$ ) isoforms in TGF- $\beta$ transgenic mice. J Am Soc Nephrol 10: 271-280, 1999.

6. Zhang $\mathrm{C}$ and Zhang F: The multifunctions of WD40 proteins in genome integrity and cell cycle progression. J Genomics 3: 40-50, 2015.

7. Kiely PA, Sant A and O'Connor R: RACK1 is an insulin-like growth factor 1 (IGF-1) receptor-interacting protein that can regulate IGF-1-mediated Akt activation and protection from cell death. J Biol Chem 277: 22581-22589, 2002.

8. Berns H, Humar R, Hengerer B, Kiefer FN and Battegay EJ: RACK1 is up-regulated in angiogenesis and human carcinomas. FASEB J 14: 2549-2558, 2000.

9. Battaini F, Pascale A, Paoletti R, Govoni S and Battaini F: The role of anchoring protein RACK1 in PKC activation in the ageing rat brain. Trends Neurosci 20: 410-415, 1997.

10. Qian L, Shi J, Zhang C, Lu J, Lu X, Wu K, Yang C, Yan D, Zhang C, You Q, et al: Downregulation of RACK1 is associated with cardiomyocyte apoptosis after myocardial ischemia/reperfusion injury in adult rats. In Vitro Cell Dev Biol Anim 52: 305-313, 2016.

11. Hu F, Tao Z, Wang M, Li G, Zhang Y, Zhong H, Xiao H, Xie X and Ju M: RACK1 promoted the growth and migration of the cancer cells in the progression of esophageal squamous cell carcinoma. Tumor Biol 34: 3893-3899, 2013.

12. Jia D, Duan F, Peng P, Sun L, Liu X, Wang L, Wu W, Ruan Y and $\mathrm{Gu}$ J: Up-regulation of RACK1 by TGF- $\beta 1$ promotes hepatic fibrosis in mice. PLoS One 8: e60115, 2013.

13. Sato M, Muragaki Y, Saika S, Roberts AB and Ooshima A: Targeted disruption of TGF- $\beta 1 / \mathrm{Smad} 3$ signaling protects against renal tubulointerstitial fibrosis induced by unilateral ureteral obstruction. J Clin Invest 112: 1486-1494, 2003.

14. Zhou P, Shi L, Li Q and Lu D: Overexpression of RACK1 inhibits collagen synthesis in keloid fibroblasts via inhibition of transforming growth factor- $\beta 1 /$ Smad signaling pathway. Int J Clin Exp Med 8: 15262-15268, 2015.

15. Blom IE, Goldschmeding R and Leask A: Gene regulation of connective tissue growth factor: new targets for antifibrotic therapy? Matrix Biol 21: 473-482, 2002.

16. Gao X, Li J, Huang H and Li X: Connective tissue growth factor stimulates renal cortical myofibroblast-like cell proliferation and matrix protein production. Wound Repair Regen 16: 408-415, 2008

17. Yokoi H, Mukoyama M, Nagae T, Mori K, Suganami T, Sawai K, Yoshioka T, Koshikawa M, Nishida T, Takigawa M, et al: Reduction in connective tissue growth factor by antisense treatment ameliorates renal tubulointerstitial fibrosis. J Am Soc Nephrol 15: 1430-1440, 2004.

18. Xiao Y, Liu J, Peng Y, Xiong X, Huang L, Yang H, Zhang J and Tao L: GSTA3 attenuates renal interstitial fibrosis by inhibiting TGF-beta-induced tubular epithelial-mesenchymal transtion and fibronection expression. PLoS One 11: e0160855, 2016.

19. Wei MG, Sun W, He WM, Ni L and Yang YY: Ferulic acid attenuates TGF- $\beta 1$-induced renal cellular fibrosis in NRK-52E cells by inhibiting Smad/ILK/Snail pathway. Evid Based Complement Alternat Med 2015: 619720, 2015. 
20. Samarakoon R, Overstreet JM, Higgins SP and Higgins PJ: TGF- $\beta 1 \rightarrow$ SMAD/p53/USF2 $\rightarrow$ PAI-1 transcriptional axis in ureteral obstruction-induced renal fibrosis. Cell Tissue Res 347: $117-128,2012$

21. Murphy M, Docherty NG, Griffin B, Howlin J, McArdle E, McMahon R, Schmid H, Kretzler M, Droguett A, Mezzano S, et al: IHG- 1 amplifies TGF- $\beta 1$ signaling and is increased in renal fibrosis. J Am Soc Nephrol 19: 1672-1680, 2008.

22. Huang C, Shen S, Ma Q, Chen J, Gill A, Pollock CA and Chen XM: Blockade of KCa3.1 ameliorates renal fibrosis through the TGF- $\beta 1 /$ Smad pathway in diabetic mice. Diabetes 62: 2923-2934, 2013.

23. Han Y, Lu JS, Xu Y, Zhang L and Hong BF: Rutin ameliorates renal fibrosis and proteinuria in 5/6-nephrectomized rats by anti-oxidation and inhibiting activation of TGF $\beta 1$-smad signaling. Int J Clin Exp Pathol 8: 4725-4734, 2015.

24. Shi Y and Massagué J: Mechanisms of TGF- $\beta$ signaling from cell membrane to the nucleus. Cell 113: 685-700, 2003.
25. Hwang M, Kim HJ, Noh HJ, Chang YC, Chae YM, Kim KH, Jeon JP, Lee TS, Oh HK, Lee YS, et al: TGF- $\beta 1$ siRNA suppresses the tubulointerstitial fibrosis in the kidney of ureteral obstruction. Exp Mol Pathol 81: 48-54, 2006.

26. Schnaper HW, Jandeska S, Runyan CE, Hubchak SC, Basu RK, Curley JF, Smith RD and Hayashida T: TGF-beta signal transduction in chronic kidney disease. Front Biosci (Landmark Ed) 14: 2448-2465, 2009.

27. Liu N, Tolbert E, Pang M, Ponnusamy M, Yan H and Zhuang S: Suramin inhibits renal fibrosis in chronic kidney disease. J Am Soc Nephrol 22: 1064-1075, 2011.

28. Vindevoghel L, Lechleider RJ, Kon A, de Caestecker MP, Uitto J, Roberts AB and Mauviel A: SMAD3/4-dependent transcriptional activation of the human type VII collagen gene (COL7Al) promoter by transforming growth factor $\beta$. Proc Natl Acad Sci USA 95: 14769-14774, 1998.

29. Lan HY: Diverse roles of TGF- $\beta /$ Smads in renal fibrosis and inflammation. Int J Biol Sci 7: 1056-1067, 2011. 